\title{
Tratamento de fobia específica por dessensibilização e reprocessamento por meio dos movimentos oculares
}

\author{
Catarina de Castro Lopes' \\ Sónia Cortinhas Carvalho \\ Maria Raquel Barbosa \\ Universidade do Porto, Porto - Portugal
}

\begin{abstract}
Resumo: A fobia específica é caracterizada pela presença de um medo persistente e recorrente que é excessivo e irracional a um objeto ou situação específica que desencadeia uma forte reação de ansiedade. Diversos estudos têm sido realizados com intuito de avaliar a eficácia da dessensibilização e reprocessamento através dos movimentos oculares (EMDR), e tem sido comprovado que é um método bem-sucedido para tratamento de fobia específica, ansiedade, depressão, traumas e queixas somáticas. $O$ caso do presente artigo descreve o trabalho terapêutico realizado com uma mulher de 42 anos com fobia a próteses dentárias através da intervenção EMDR. No final da intervenção, a paciente perdeu o medo às próteses, já não revelando respostas de ansiedade e de desconforto, mantendo-se estável durante o período de follow-up, um mês após a intervenção. Esses resultados repercutiram-se na melhoria da sua qualidade de vida, ao nível da sua vida pessoal, social e profissional.
\end{abstract}

Palavras-chave: fobia específica; estudo de caso; trauma; ansiedade; movimentos oculares.

\section{TREATMENT OF SPECIFIC PHOBIA THROUGH EYE MOVEMENT DESENSITIZATION AND REPROCESSING}

\begin{abstract}
The specific phobia is characterized by a persistent and recurring fear that is excessive and irrational to a specific object or situation, triggering a strong anxiety reaction. Several studies have been conducted in order to assess the effectiveness of eye movement desensitization and reprocessing (EMDR) and it has been proven to be a successful method for the treatment of specific phobias, anxiety, depression, trauma and somatic complaints. The case of this article describes the therapeutic work done with a forty-two years old' woman that has phobia to dentures using the intervention of EMDR. At the end of the intervention, the patient lost the fear of prosthetics, no longer revealing responses of anxiety and discomfort and remained stable during the follow-up one month after the intervention. These results had an impact on improving their quality of life, in terms of her personal, social and professional life.
\end{abstract}

Keywords: specific phobia; case study; trauma; anxiety; eye movement.

\section{TRATAMIENTO DE LA FOBIA ESPECÍFICA POR DESENSIBILIZACIÓN Y REPROCESAMIENTO A TRAVES DE LOS MOVIMIENTOS OCULARES}

Resumen: La fobia específica se caracteriza por la presencia de un miedo persistente y recurrente que es excesivo o irracional a un objeto o situación especifica que desencadena

1 Endereço para correspondência: Catarina de Castro Lopes, Departamento de Psicologia Clínica, Clínica White, Rua Dr. António Loureiro Borges, Edificio 5, $1^{\circ}$ andar, Arquipark, Miraflores, Algés - Portugal. CEP: 1495-131. E-mail: catarinacastrolopes@white.pt. 
una fuerte reacción de ansiedad. Varios estudios han sido realizados con el fin de evaluar la eficacia de desensibilización y reprocesamiento mediante estudio de los movimientos oculares (EMDR) y ha sido comprobado que es un método exitoso para el tratamiento de la fobia específica, ansiedad, depresión, traumas y quejas somáticas. El presente artículo describe el trabajo terapéutico realizado con una mujer de cuarenta y dos años con fobia a prótesis dentarias a través de la intervención EMDR. En el final de la intervención, la paciente perdió el miedo a las prótesis, no evidenciando señales de ansiedad y de desconforto, manteniéndose estable durante el periodo de follow-up, un mes después de la intervención. Eses resultados repercutieran en una notable mejoría de su calidad de vida a nivel personal, social y profesional.

Palabras clave: fobia específica; estudio de caso; trauma; ansiedad; movimientos oculares.

De acordo com o Manual de diagnóstico e estatística das perturbações mentais: DSM-IV-TR (American Psychiatric Association, 2006), a fobia específica é caracterizada pela presença de medo acentuado e persistente que é excessivo ou irracional, desencadeado pela presença ou antecipação de um objeto ou situação específica. A exposição ao estímulo fóbico provoca quase invariavelmente uma resposta ansiosa imediata. A pessoa reconhece que o medo é excessivo, no entanto não tem controlo sobre suas reações. As situações fóbicas são evitadas com intensa ansiedade e mal-estar, interferindo significativamente na rotina diária, no funcionamento ocupacional, nos relacionamentos interpessoais e nas atividades sociais da pessoa. $O$ evitamento fóbico é a consequência mais complicada devido ao impacto que tem na vida da pessoa com limitação da sua liberdade individual.

Os primeiros sintomas de fobia específica ocorrem habitualmente na infância ou no início da adolescência, podendo, porém, ocorrer mais cedo nas mulheres do que nos homens. Os fatores predisponentes para o desencadeamento da fobia incluem, regra geral, acontecimentos traumáticos que tendem a ter um desenvolvimento agudo (American Psychiatric Association, 2006).

Nesta esteira, a dessensibilização e reprocessamento através dos movimentos oculares (eye movement desensitization and reprocessing - EMDR) tem sido indicada, nos últimos anos, como tratamento para problemas de ansiedade, incluindo post-traumatic stress disorder (PTSD) e várias fobias. Desenvolvida originalmente, em 1989, por Francine Shapiro, uma psicóloga americana, a EMDR é considerada atualmente um tratamento eficaz para situações traumáticas por algumas organizações, como sejam a American Psychiatric Association (2004) e o National Institute for Health and Clinical Excellence (2006). As mais recentes avaliações por parte da International Society for Traumatic Stress (Foa, Keane, Friedman, \& Cohen, 2009) e do Department of Veterans Affairs and Department of Defense (2010) dos Estados Unidos designam a EMDR como um tratamento de nível $\mathrm{A}$, descrito por este último como uma excelente recomendação a nível interventivo que os médicos podem fornecer para alguns pacientes.

A EMDR ajuda a desbloquear o sistema nervoso, permitindo que o cérebro processe a experiência traumática. $O$ terapeuta executa a estimulação bilateral que favorece a comunicação entre os dois hemisférios cerebrais, através de movimentos laterais oculares, sons alternados, ou toques nas mãos ou joelhos, enquanto o cliente acede 
simultaneamente à memória traumática armazenada mediante as imagens, cognições, emoções e sensações (Wesselmann et al., 2012). É um processo semelhante ao que se passa quando sonhamos na chamada fase REM do sono, durante a qual os movimentos oculares rápidos facilitam o processamento do material inconsciente. Quando surge uma situação traumática, esta pode ficar bloqueada no sistema nervoso da pessoa com a recordação original, os sons, os pensamentos, as emoções do passado e as sensações físicas. A intervenção EMDR permite que a pessoa identifique, e separe, as sensações afetivas do trauma das suas interpretações cognitivas, ajudando a dessensibilizar as memórias traumáticas (Luber, 2009).

A EMDR é uma abordagem de oito etapas específicas que move o cliente por meio de conceptualização de caso e preparação do protocolo, a dessensibilização e o reprocessamento da memória traumática, fecho e follow-up (Wesselmann et al., 2012). Ao longo do processo de oito fases, são contemplados os protocolos para eventos passados que estabelecem as bases para a patologia, as situações actuais que causam perturbação e futuros modelos para a acção futura apropriada ou adequada (Shapiro, 2005).

No estudo de Van der Kolk et al. (2007), a EMDR provou ser mais bem-sucedida do que a farmacoterapia para alcançar melhorias significativas na PTSD e nos sintomas depressivos, em sobreviventes de trauma. Contudo, algumas meta-análises (Bisson et al., 2007; Etten \& Taylor, 1998) indicam que a EMDR consegue efeitos terapêuticos que são equivalentes e tão duradouros como os dos métodos da abordagem cognitivo-comportamentais. Ainda assim, a EMDR tem sido usada para melhorar a regulação afetiva e alterar características de personalidade (Brown \& Shapiro, 2006), diminuir a dor do membro fantasma (Schneider, Hofmann, Rost, \& Shapiro, 2008) e as queixas somáticas (Gupta \& Gupta, 2002), melhorar a depressão em adolescentes (Bae, Kim, \& Park, 2008) e tratar de problemas de ansiedade, como sejam fobias, perturbação de pânico e perturbação de ansiedade generalizada (De Jongh, Van den Oord, \& Ten Broeke, 2002; Fernandez \& Faretta, 2007; Gauvreau \& Bouchard, 2008).

A principal vantagem desta abordagem terapêutica sobre a clássica terapia cognitivo-comportamental é a rapidez da sua ação e menor número de sessões envolvidas (De Jongh \& Ten Broeke, 1998, 2007; De Jongh et al., 2002; Roos \& De Jongh, 2008). Por exemplo, no caso da fobia de aranhas, as pessoas são expostas ao animal e encorajadas a deixar as aranhas andar pelas suas mãos. As técnicas de exposição in vivo têm realmente mostrado eficácia neste tipo de fobias. Contudo, existem poucos estudos que se centram na avaliação da eficácia das técnicas de exposição em casos de fobias originadas por episódios traumáticos (como acidentes de carro, medo de engasgar, mordidelas de cão). De fato, as pessoas não podem ser expostas a mordidelas de cães ou acidentes de viação, entretanto a antecipação destes eventos pode tornar as situações temíveis (De Jongh \& Ten Broeke, 1998, 2007).

De Jongh, Ten Broeke e Renssen (1999) referem que algumas fobias específicas, tais como medo de cães, sangue, injeções, ferimentos, falar em público e fobia dentária, habitualmente têm origem numa experiência traumática. Segundo os mesmos autores, 
a EMDR é consideravelmente mais eficaz, eficiente e confortável para o paciente do que as técnicas de exposição. De Jongh et al. (1999) trabalharam com pacientes com fobia dentária e verificaram que a EMDR é consideravelmente mais eficaz, eficiente e confortável para o paciente do que as técnicas de exposição, principalmente quando estão envolvidas experiências traumáticas. Neste sentido, Marcus, Marquis e Saki (1997), num estudo com indivíduos com PTSD, compararam a terapia EMDR com o acompanhamento tradicional (por exemplo, terapia cognitivo-comportamental, psicoterapia psicodinâmica, medicação e terapia de grupo), e foi apurado que os pacientes tratados com EMDR mostraram melhoria significativa dos sintomas e duas vezes mais rápida do que o acompanhamento tradicional.

O protocolo EMDR ajuda a dessensibilizar as memórias traumáticas, cujo trabalho terapêutico baseia-se na imaginação do estímulo fóbico acompanhado de estimulação bilateral - ocular, auditiva ou toque (De Jongh et al., 1999; Shapiro, 2002).

Se a ansiedade do paciente face ao objeto é causada por uma situação traumática, deve-se avançar com o processamento EMDR. Se o cliente é estruturalmente ansioso ou tem uma vinculação insegura, então talvez seja necessário usar algumas intervenções antes do processamento EMDR, como a instalação do lugar seguro. Algumas pessoas mais ansiosas têm dificuldade em encontrar um lugar seguro. Às vezes mudar o nome ajuda. Pode ser um lugar mais confortável ou lugar relaxante. Pode ajudar também nestes casos fazer treino de relaxamento, como relaxamento muscular progressivo ou respiração diafragmática (Shapiro, 2002). Essa instalação de recurso é útil para apoiar o trabalho de reprocessamento que pode ser desgastante em alguns casos, antes de o iniciarem ou durante o trabalho, pois ajuda a ativar, reforçar e facilitar o acesso a qualidades, emoções e crenças - recursos, na linguagem EMDR - que sejam necessárias para que o processamento possa continuar com tranquilidade. Ajuda a preparar o paciente para o trabalho de trauma e a desenvolver forças e competências (Shapiro, 2009).

Outra estratégia que ajuda no processo de processamento é o uso de entrelaçamento cognitivo que são afirmações ou questões que o terapeuta faz ao paciente para ajudar a desbloquear quando processo está em looping (sem mudanças na informação a que acede), quando existem muitas generalizações ou muito stresse emocional (dissociação). Estas afirmações ou questões feitas pelo terapeuta têm como intenção aceder à informação que o paciente possui, mas não está disponível na rede de memória em que se encontra, ou, pelo contrário, pode ter como objetivo oferecer informação que o paciente não tem, mas que pode ajudar a estimular conexões para que o reprocessamento continue (Shapiro, 2002).

Neste âmbito, é descrito um caso clínico de uma mulher - Maria (nome fictício) que apresentava fobia específica a objetos em forma de dentes ou dentaduras, cujo objetivo é o de analisar a eficácia da EMDR no caso de fobia específica. A seguir, apresentam-se a descrição clínica do caso, os procedimentos de avaliação e intervenção, os resultados e a respetiva discussão. 


\section{Método}

\section{Caracterização clínica do caso}

Maria tem 42 anos de idade, é divorciada, sem filhos, com estatuto socioeconómico baixo, vive sozinha e trabalha num supermercado. Exibe fobia específica a próteses dentárias desde os 6 anos, desencadeada por experiências traumáticas que teve no passado. A exposição ao objeto ou o simples fato de imaginar a prótese provocava uma resposta de intensa ansiedade, cujos sintomas mais evidentes e desconfortáveis refletiam-se na sensação de falta de ar, tremores, sudação, choro, rubor facial e aceleração do batimento cardíaco. Este medo era persistente e manifestava-se de uma forma excessiva, desadaptativa e descontrolada, provocada pela presença ou antecipação da prótese.

A paciente tinha consciência de que o seu medo era desproporcional e exagerado face à situação, visto que a prótese era um objeto e não lhe iria fazer mal, mas, mesmo assim, não conseguia controlar as suas reações. Este quadro sintomático interferia com a sua rotina diária, induzindo-lhe bastante sofrimento. Ao nível das relações interpessoais, evitava encontros sociais, nomeadamente jantares e festas, se soubesse previamente que iriam estar presentes pessoas com próteses removíveis, devido ao medo antecipatório de que a prótese pudesse cair ou mover-se. No contexto profissional, pelas mesmas razões, não ia almoçar com ninguém. Na vida familiar, ela fugia dos seus irmãos, porque as brincadeiras preferidas deles consistiam em lhe despertar o medo através de objetos em forma de dentaduras e dentes, inclusive com as suas próprias próteses removíveis. Também descurava a sua saúde dentária, evitando consultar um médico dentista, e, por conseguinte, a sua falta de dentes interferia na capacidade de mastigação e no seu bem-estar, razão pela qual procurou intervenção psicológica.

Tudo começou quando tinha 6 anos e viu a mãe a retirar a prótese removível para a lavar (nas mãos). A interpretação dada a este momento - "a boca está na mão, está no sítio errado" - despoletou emoções e sensações de nojo, medo e repulsa que a levavam a fugir e a isolar-se no quarto a chorar. Contudo, a paciente sempre teve uma boa relação com a mãe e com o resto da família. Não se recorda em concreto o que sentiu relativamente à mãe naquela altura, mas pensa que terá sido um sentimento de pena por ela não ter dentes e precisar de prótese dentária. Na altura (aos 6 anos), não falou acerca deste episódio com a mãe, pois não queria magoá-la perante a ideia de que "a sua boca estava no sítio errado". Só por volta dos 10 anos (quando que viu novamente a prótese) assumiu a sua fobia e contou à mãe. A resposta que recebeu dela foi que a sua prótese era um objeto e não lhe faria mal, sendo o medo dela "estúpido".

Maria tinha como objetivo mudar a sua aparência dentária, e a solução era a colocação de uma prótese dentária. Contudo, qualquer objeto em forma de dentes ou semeIhante à prótese dentária, como dentaduras, coroas ou moldes dentários, causava-lhe resposta de intensa ansiedade. Já tinha feito uma tentativa para tratar da sua saúde e estética oral com ajuda de ansiolíticos receitados pelo seu médico de família, mas não obteve qualquer sucesso. Foi impossível conseguir fazer os moldes para colocação de 
prótese dentária, e, portanto, não pôde fazer o tratamento. Por esta razão, ela sentiu a necessidade de pedir apoio psicológico, não só para conseguir executar o tratamento dentário, mas também para melhoria do seu bem-estar psicológico.

Optou-se pela intervenção EMDR, uma vez que, na origem desta fobia, estava um episódio de trauma na infância. Maria foi informada sobre a terapia EMDR e deu consentimento para a utilização do procedimento.

\section{Instrumentos}

A paciente foi avaliada psicologicamente através do questionário The Symptom Checklist-90 (SCL-90) e entrevista de avaliação clínica. As informações obtidas através do SCL-90 apontaram para a presença de perturbação de ansiedade, e, mediante a entrevista individual, pôde-se perceber que se tratava de fobia específica. Todos os critérios de diagnóstico, segundo o Manual de diagnóstico e estatística das perturbações mentais: DSM-IV-TR (American Psychiatric Association, 2006, p. 449) estavam presentes.

No transcorrer do processo de intervenção, usaram-se as escalas validity of cognitive (VOC), para avaliar a validade da cognição positiva, e subjective unit of distress (SUD), para avaliar o stresse.

\section{Procedimento}

Baseando-se no protocolo de EMDR, as situações emocionalmente difíceis do passado foram reprocessadas, bem como os estímulos atuais que provocavam medo a Maria, através de estimulação bilateral ocular, para que ela conseguisse estar perante os objetos indutores de ansiedade. No início do trabalho terapêutico, foram reprocessados os eventos que contribuíram para fobia, a primeira vez que o medo foi experienciado, as experiências mais perturbadoras, a última vez que sentiu medo, as associações cognitivas e sensações físicas aferentes a objetos em forma de dentes ou dentaduras. A instalação de recursos e os entrelaçamentos cognitivos usados a partir da sexta sessão afiguraram-se técnicas poderosas que proporcionaram um grande avanço no processo terapêutico. As sessões tiveram periodicidade semanal, tendo cada uma a duração de uma hora. A seguir, apresenta-se o protocolo de EMDR posto em prática:

- Primeira sessão: a situação escolhida (recordação original) foi a mãe lavar as próteses quando a paciente tinha 6 anos. A imagem escolhida para representar o momento mais difícil foi "a mãe ter a boca na mão". A cognição negativa (CN) tinha a ver com a possibilidade de escolha/controlo: "Eu sou fraca". Gostava de substituir esta CN pela cognição positiva (CP): "Eu sou forte e capaz de ultrapassar este medo estúpido". Quando pensava na imagem e na CP, numa escala de um a sete (VOC), atribuiu valor um (valor mais baixo). As emoções sentidas no momento foram: medo, repulsa e nojo. O seu stresse foi avaliado através do instrumento SUD, escala que varia de zero a dez, tendo atribuído o valor máximo. As sensações corporais relatadas foram pressão/aperto na boca do estômago, no peito (que a impediam de respirar normalmente) e nos braços, nó na garganta, tremores, formigueiro em 
braços e pernas, sensação de tendões puxados. Foi instalado o lugar seguro: no Gerês, no campo em contacto com os animais, que chamava "paraíso"; assim conseguiu entrar em contacto com sensações de calma e tranquilidade.

- Sessões seguintes (da segunda à quinta sessão): depois de completada a fase de preparação e avaliação, procedeu-se à dessensibilização. Durante esta fase, Maria refugiava-se no seu lugar seguro (campo rodeado de animais), usando recorrentemente essa estratégia para lidar com as memórias perturbadoras que iam surgindo durante o processamento. Também se recordou de algumas situações que estavam esquecidas, almoços e jantares com amigos, do aniversário da avó em que viu a placa saltar quando soprava as velas do bolo, lembrou-se ainda de, num museu de dinossauros, ter visto maxilares e dentes. O que foi peculiar durante o tratamento foi o surgimento de "memórias de outras vidas", segundo as palavras da paciente, nas quais era uma enfermeira em tempo de guerra que assistiu a torturas altamente violentas, em que eram arrancados maxilares às pessoas e colocados num frasco de vidro. O aparecimento deste material talvez se possa justificar pelo fato de ela acreditar em vidas passadas. Não obstante a importância de todo o material surgido até aqui ("vidas passadas" e "vida atual"), o processo ficou em looping. Ou seja, não aconteciam mudanças no processamento de informação, ela oscilava entre as memórias passadas relacionadas com dentes ou dentaduras e o lugar seguro.

- Sexta sessão: foi feita a instalação de recursos, tendo a paciente escolhido ter controlo sobre si e suas emoções. Instalar a imagem futura de estar perante o objeto fóbico acompanhada pelo pensamento de ter controlo sobre si própria foi bastante útil e permitiu que a paciente avançasse bastante o processo. Neste sentido, a paciente escolheu usar uma memória particular "a trabalhar no supermercado" que despoletou emoções e sensações de orgulho, profissionalismo, controlo e força. Ainda nesta sessão, a paciente obteve outra vitória, tendo conseguido visualizar gomas em forma de dentes a dirigirem-se a si e gritou "vitória!" quando conseguiu comer uma dessas gomas. Além deste entrelaçamento cognitivo, outros se revelaram eficazes: reforçar que Maria era uma criança na altura e perguntar-lhe o que ela pensava sobre a situação enquanto adulta; pedir-lhe que imaginasse por um segundo que era forte e capaz de ultrapassar o seu medo; uso do método socrático.

- Sétima sessão: nesta sessão, como forma de fornecer ferramentas de auto-controlo para lidar com o medo, foi usada a técnica light stream. Pediu-se à paciente que atribuísse forma, tamanho, cor, temperatura, textura e som às sensações e tensões que lhe percorriam todo o corpo. Rapidamente, ela chegou à conclusão de que se tratava de uma luz quente, inflamável de cor vermelha que atravessava o seu corpo, atribuindo o nome de "vírus". De seguida, perguntou-se-lhe qual seria a cor, forma e textura associada ao bem-estar para ajudar a combater esse vírus. A paciente respondeu que seria uma luz branca, agradável em forma de círculo, flexível e forte à qual chamou "antibiótico". Durante o processamento, o "antibiótico" começou a ganhar cada vez mais poder e a combater o vírus, até que Maria conseguiu verbalizar "Yes! Antibiótico venceu o vírus!". 
- Oitava sessão: o sentimento que surgiu quando se recordou da situação foi a pena da mãe por não ter dentes naturais, referindo que já não a perturbava quase nada. Nesta fase, o valor de SUD foi de dois, e referiu sentir no corpo apenas um formigueiro. Pediu-se-lhe que visualizasse a sequência de eventos ao longo do tempo e espaço como se de um filme se tratasse. A SUD ficou igual a zero, e assim avançamos para a instalação de recursos até o valor da escala VOC atingir sete valores. Nesta altura, a paciente afirmou sentir as palavras como totalmente verdadeiras: "Eu sou forte e capaz de ultrapassar este medo estúpido" (CP). Tornou-se a fazer a revista de todo o seu corpo (sonda corporal) e não revelou qualquer sensação desconfortável.

- Nona sessão: foram trabalhados cenários futuros, tais como ver alguém com prótese, o irmão a brincar com ela, cair a prótese de alguém durante o jantar, fazer moldes para tratamento dentário, colocar uma prótese em boca. Desta forma, foi instalada a CP até o valor da escala VOC atingir sete valores.

- Décima sessão: a paciente parecia preparada para confrontar a situação in vivo. Quando se lhe pediu para imaginar uma prótese, o seu nível de stresse (SUD) foi igual a zero, não surgindo qualquer sensação ou emoção desconfortável. Nesta última sessão, numa primeira fase, ela começou por observar a prótese e não sentiu qualquer desconforto, e depois foi-lhe pedido para pegar na prótese, ficando com ela na mão, e a SUD permaneceu igual a zero.

\section{Resultados}

Em dez sessões de EMDR, Maria perdeu o medo das próteses. Atualmente, já consegue visualizar e até pegar em dentaduras, próteses e objetos em forma de dentes sem apresentar qualquer resposta de ansiedade ou medo e, inclusive, já colocou uma prótese dentária, tendo completado o seu tratamento dentário com sucesso. O médico dentista que a tratou ficou surpreendido com a sua cooperação.

Durante o período de follow-up, um mês após intervenção, foi mostrada a prótese, e a paciente voltou a tocar-lhe, o que se revelou favorável, isto é, as reações e sensações em relação ao objeto prótese mantiveram-se (ausência de ansiedade ou desconforto). Em análise, ela referiu ter melhor qualidade de vida, que se reflete na sua vida pessoal, familiar, profissional e social, pois já não evita nenhuma situação nem o fato de estar com pessoas que usem prótese removível. A sua capacidade de mastigação é bastante satisfatória e já não descura a sua saúde dentária, indo ao dentista assiduamente. Referiu ainda conseguir gerir melhor qualquer situação de ansiedade, recorrendo com frequência ao seu lugar seguro.

\section{Discussão}

O caso clínico descrito no presente artigo apresenta uma fobia específica com origem numa experiência traumática. Segundo a American Psychiatric Association (2006), 
os factores predisponentes para o desencadeamento de fobia incluem, geralmente, acontecimentos traumáticos.

O protocolo usado ajudou a dessensibilizar e a reprocessar as memórias traumáticas, resultando na redução da ansiedade, em alterações cognitivas e comportamentos mais adaptativos. Esta situação corrobora a literatura que aponta para a eficácia da EMDR no tratamento de problemas afins (De Jongh \& Ten Broeke, 1998; De Jongh et al., 2002; Roos \& De Jongh, 2008; Schurmans, 2007; Shapiro, 2002).

Por exemplo, De Jongh e Ten Broeke (1998) realizaram um estudo de caso da aplicação da terapia EMDR com uma mulher que apresentava medo de sufocar ou asfixiar, o que lhe impedia de realizar tratamentos dentários. A paciente tinha tido uma experiência traumática cinco anos antes do início da terapia que lhe provocou a fobia. Tinha planeado um tratamento para alinhar os dentes, no entanto assumia-se incapaz por causa do medo extremo de asfixia. Os resultados da terapia EMDR em apenas duas sessões (cada uma com menos de uma hora de tempo de tratamento) foram bastante consistentes. Teve um efeito generalizado sobre o seu medo, tornando possível engolir alimentos normalmente, e essas mudanças mantiveram-se estáveis após 12 meses de follow-up.

Existem outros exemplos na literatura científica que corroboram a eficácia da EMDR também em casos clínicos de fobia específica. Roos e De Jongh (2008) ajudaram, através desta terapia, quatro crianças e adolescentes entre os 3 e os 15 anos que apresentavam fobia de asfixia de natureza traumática. Os resultados evidenciaram-se logo após duas sessões - as queixas relacionadas com a deglutição diminuíram, assim como a fadiga e a tristeza -, o que levou à conclusão de que a EMDR é um método eficaz. De forma similar, Schurmans (2007) usou a EMDR com uma mulher com a mesma fobia que anteriormente já tinha recebido tratamento durante quatro anos através de terapia psicodinâmica breve, cognitivo-comportamental e tratamento farmacológico. Os resultados desses tipos de tratamento assumiram-se improfícuos. Em decorrência da aplicação da EMDR, denotou-se a remissão dos sintomas, tendo sido possível conferir uma nova interpretação à situação e memórias traumáticas que despoletaram sensações de segurança e conforto emocional.

O uso da EMDR no nosso caso clínico mostrou-se eficaz em apenas dez sessões, indo ao encontro de várias pesquisas que conseguiram resultados eficazes para o tratamento de fobia especifica em poucas sessões (De Jongh \& Ten Broeke, 1998, 2007; De Jongh et al., 2002; Roos \& De Jongh, 2008), o que reforça a sua vantagem relativamente a outras terapias, nomeadamente a cognitivo-comportamental (comumente usada), não só pelo menor número de sessões envolvidas, o que naturalmente leva a que seja um tratamento menos dispendioso, como pelo maior conforto que proporciona ao paciente, uma vez que não necessita de exposição in vivo. Embora fosse possível recorrer à exposição in vivo no nosso caso clínico, expondo a paciente a uma dentadura ou outro objecto em forma de dentes, esse tipo de intervenção provocaria ansiedade antecipatória e bastante sofrimento durante a exposição, pois estaria em contato com o medo fóbico (De Jongh \& Ten Broeke, 1998; De Jongh et al., 1999). 
Verificou-se ainda que a terapia EMDR provou ser mais eficaz do que a farmacoterapia, uma vez que a paciente já tinha recorrido a ajuda farmacológica (ansiolíticos) para tentar fazer o seu tratamento dentário, o que vai ao encontro dos resultados da pesquisa de Van Der Kolk et al. (2007).

De acordo com os mesmos autores, as melhorias com a terapia vão além dos sintomas evidentes provocados pela situação traumática. Maria, além de ter melhorado a sua higiene oral, melhorou a sua qualidade de vida e adquiriu recursos para regular a sua ansiedade, referindo usar o lugar seguro como forma de se acalmar.

A instalação de recursos revelou-se muito útil, tendo preparado Maria para o processamento da EMDR, fornecendo-lhe ferramentas para lidar com memórias traumáticas e adquirir novas aprendizagens, indo ao encontro das constatações de Shapiro (2009) e Luber (2009). Por sua vez, o entrelaçamento cognitivo ajudou a desbloquear o processamento EMDR, tendo ajudado Maria a dar novas interpretações as suas memórias, o que corrobora o descrito na literatura (Shapiro, 2009).

A limitação do caso descrito neste artigo é que não foi usada nenhuma medida de avaliação padronizada. Além disso, o caso apresentado não pode ser representativo da população com fobia específica. O exemplo é insuficiente para tirar conclusões gerais sobre a eficácia da EMDR com estas perturbações. Tal como De Jongh e Ten Broeke (1998) referem, o suporte empírico para a aplicação da EMDR com fobias específicas é escasso, sendo necessária mais investigação nesta área, particularmente estudos comparativos entre a terapia cognitivo-comportamental com a exposição in vivo e a EMDR para fobia específica. Seria interessante comparar a exposição imagética utilizada na terapia cognitivo-comportamental com EMDR em casos de fobias, em que não é possível usar a exposição in vivo (por exemplo, medo de ter um acidente).

Espera-se que com o presente artigo se compreenda melhor a intervenção assente na EMDR e a sua importância em casos de fobias. É um poderoso e surpreendente método terapêutico, pois, em pouco tempo, os sintomas de que os pacientes se queixam desaparecem e as situações que eram inicialmente perturbadoras deixam de o ser, o que se prolonga no tempo. Outra grande vantagem é que, ao trabalharmos sobre um problema específico, podemos estar a trabalhar outras dificuldades do paciente em simultâneo, uma vez que se está a trabalhar a atribuição de novos sentidos, mais positivos, às experiências traumáticas. A EMDR pode ser uma alternativa eficaz para o tratamento de condições fóbicas com uma etiologia traumática.

\section{Referências}

American Psychiatric Association. (2004). Practice guideline for the treatment of patients with acute stress disorder and post-traumatic stress disorder. Arlington: American Psychiatric Association Practice Guidelines.

American Psychiatric Association. (2006). Manual diagnóstico e estatística das perturbações mentais: DSM-IV-TR (J. N. Almeida, Trad.) Lisboa: Climepsi Editores (Obra original publicada em 2000). 
Bae, H., Kim, D., \& Park, Y. C. (2008). Eye movement desensitization and reprocessing for adolescent depression. Psychiatry Investigation, 5, 60-65.

Bisson, J., Ehlers, A., Matthews, R., Pilling, S., Richards, D., \& Turner, S. (2007). Psychological treatments for chronic post-traumatic stress disorder: systematic review and meta-analysis. The British Journal of Psychiatry, 190, 97-104.

Brown, S., \& Shapiro, F. (2006). EMDR in the treatment of borderline personality disorder. Clinical Case Studies, 5, 403-420.

De Jongh, A., \& Ten Broeke, E. (1998). Treatment of choking phobia by targeting traumatic memories with EMDR: a case study. Clinical Psychology and Psychotherapy, 5, 264-269.

De Jongh, A., \& Ten Broeke, E. (2007). Treatment of specific phobias with EMDR: conceptualization and strategies for the selection of appropriate memories. Journal of EMDR Practice and Research, 1(1), 46-57.

De Jongh, A., Ten Broeke, E., \& Renssen, M. R. (1999). Treatment of specific phobias with eye movement desensitization and reprocessing (EMDR): protocol, empirical status, and conceptual issues. Journal of Anxiety Disorders, 1-2(13), 69-85.

De Jongh, A., Van den Oord, H. J., \& Ten Broeke, E. (2002). Efficacy of eye movement desensitization and reprocessing (EMDR) in the treatment of specific phobias: four single-case studies on dental phobia. Journal of Clinical Psychology, 58(12), 1489-1503.

Department of Veterans Affairs and Department of Defense. (2010). Clinical practice guideline for the management of post-traumatic stress. Washington: Veterans Health Administration, Department of Veterans Affairs and Health Affairs, Department of Defense.

Etten, M., \& Taylor, S. (1998). Comparative efficacy of treatments for post-traumatic stress disorder: a meta-analysis. Clinical Psychology and Psychotherapy, 5, 126-144.

Fernandez, I., \& Faretta, E. (2007). EMDR in the treatment of panic disorder with agoraphobia. Clinical Case Studies, 6, 44-63.

Foa, E. B., Keane, T. M., Friedman, M. J., \& Cohen, J. A. (2009). Effective treatments for PTSD: practice guidelines of the international society for traumatic stress studies. New York: Guilford Press.

Gauvreau, P., \& Bouchard, S. P. (2008). Preliminary evidence for the efficacy of EMDR in treating generalized anxiety disorder. Journal of EMDR Practice and Research, 2, 26-40.

Gupta, M., \& Gupta, A. (2002). Use of eye movement desensitization and reprocessing (EMDR) in the treatment of dermatologic disorders. Journal of Cutaneous Medicine and Surgery, 6, 416-421.

Luber, M. (2009). EMDR scripted protocols. New York: Spinger. 
Marcus, S. V., Marquis, P., \& Saki, C. (1997). Controlled study of treatment of PTSD using EMDR in an HMO setting. Psychotherapy, 34, 307-315.

National Institute for Health and Clinical Excellence. (2006). The public health guidance development process: an overview for stakeholders including public health practitioners, policy makers and the public. Recuperado em $1^{\circ}$ novembro, 2013, de www.nice.org.uk

Roos, C., \& De Jongh, A. (2008). EMDR treatment of children and adolescents with a choking phobia. Journal of EMDR Practice and Research, 3(2), 201-211.

Schneider, J., Hofmann, A., Rost, C., \& Shapiro, F. (2008). EMDR in the treatment of chronic phantom limb pain. Pain Medicine, 9, 76-82.

Schurmans, K. (2007). A clinical vignette - EMDR treatment of choking phobia. Journal of EMDR and Research, 2(2), 118-121.

Shapiro, F. (2002). EMDR as an integrative psychotherapy approach: experts of diverse orientations explore the paradigm prism. Washington: American Psychological Association.

Shapiro, F. (2005). EMDR solutions. Pathways to healing. New York: Norton \& Company.

Shapiro, F. (2009). The EMDR approach to psychotherapy. Watsonville: EMDR Institute.

Van der Kolk, B., Spinazzola, B., Blaustein, M. E., Hopper, J. W., Hopper, E. K., Korn, D. L., \& Simpson, W. B. (2007). A randomized clinical trial of eye movement desensitization and reprocessing (EMDR), Fluoxetine, and Pill placebo in the treatment of posttraumatic stress disorder: treatment effects and long-term maintenance. Journal of Clinical Psychiatry, 68, 1-10.

Wesselmann, D., Davidson, M., Armstrong S., Schweitzer C., Bruckner D., \& Potter A. E. (2012). EMDR as a treatment for improving attachment status in adults and children. Revue Européenne de Psychologie Appliquée, 62, 223-230.

Submissão: 25.7 .2012

Aceitação: 4.11 .2013 